\title{
A study on the transition between seniority-type and collective excitations in ${ }^{204} \mathrm{Po}$ and ${ }^{206} \mathrm{Po}$
}

\author{
Milena Stoyanova ${ }^{1, *}$, Georgi Rainovski ${ }^{1}$, Jan Jolie ${ }^{3}$, Norbert Pietralla ${ }^{2}$, Andrey Blazhev ${ }^{3}$, Martin Djongolov ${ }^{1}$, Arwin \\ Esmaylzadeh $^{3}$, Lisa Gerhard $^{3}$, Kalin Gladnishki ${ }^{1}$, Vasil Karayonchev ${ }^{3}$, James Keatings ${ }^{4}$, Ralph Kern ${ }^{2}$, Diana Kocheva ${ }^{1}$, \\ Thorsten Kröll ${ }^{2}$, Konstantin Mashtakov ${ }^{4}$, Oliver Möller ${ }^{2}$, Jean-Marc Régis ${ }^{3}$, Marcus Scheck $^{4}$, Kerstin Schomacker $^{3}$, \\ Jacqueline Sinclair ${ }^{4}$, Chistian Sürder ${ }^{2}$, Volker Werner ${ }^{2}$, and Johannes Wiederhold ${ }^{2}$
}

${ }^{1}$ Faculty of Physics, St. Kliment Ohridski University of Sofia, 1164 Sofia, Bulgaria

${ }^{2}$ Institut für Kernphysik, Technische Universität Darmstadt, 64289 Darmstadt, Germany

${ }^{3}$ Institut für Kernphysik, Universität zu Köln, 50937 Köln, Germany

${ }^{4}$ School of Engineering \& Computing, University of the West of Scotland, Paisley PA1 2BE, United Kingdom

\begin{abstract}
Low-lying yrast states in ${ }^{204} \mathrm{Po}$ and ${ }^{206} \mathrm{Po}$ have been investigated by the $\gamma-\gamma$ fast timing technique with $\mathrm{LaBr}_{3}(\mathrm{Ce})$ detectors. Excited states of these nuclei were populated in the ${ }^{197} \mathrm{Au}\left({ }^{11} \mathrm{~B}, 4 \mathrm{n}\right)$ and the ${ }^{198} \mathrm{Pt}\left({ }^{12} \mathrm{C}, 4 \mathrm{n}\right)$ fusion-evaporation reactions, respectively, at the FN-Tandem Facility at the University of Cologne. The lifetimes of the $4_{1}^{+}$states in both nuclei were measured, along with an upper limit for the $2_{1}^{+}$state in ${ }^{204}$ Po. The preliminary results are discussed in the scope of the systematic behavior of the transition strengths between yrast states in polonium isotopes.
\end{abstract}

\section{Introduction}

The evolution from seniority-type to collective structure is a process which provides a stringent test for the contemporary nuclear structure models. The yrast structures of polonium isotopes below the $N=126$ shell closure are suitable for studying this evolution. In a recent study [1], an increased strength for the seniority-changing $2_{1}^{+} \rightarrow 0_{1}^{+}$transition in ${ }^{206} \mathrm{Po}$ has been reported, leading to the conclusion that the $2_{1}^{+}$state of ${ }^{206} \mathrm{Po}$ has a predominantly collective character. It has to be noted however, that the energy level pattern of the yrast states of ${ }^{206} \mathrm{Po}$ and the hindered transition probability of the $8_{1}^{+} \rightarrow 6_{1}^{+}$transition indicate that seniority-type structure is preserved to a certain extent. This fact either implies a spin dependence in the evolution from seniority-type regime to collectivity or questions the conclusions in Ref. [1]. The later stems from the fact that in the seniority regime, the $E 2$ transition strength of seniority changing transitions, such as the $2_{1}^{+} \rightarrow 0_{1}^{+}$transition, increases in a parabolic way with increasing the number of valence particles and reaches a maximum at the middle of the $j$-shell [2]. This behavior is identical to the one in the collective regime and therefore the evolution of the $E 2$ transition strength for the $2_{1}^{+} \rightarrow 0_{1}^{+}$ transitions cannot be used as a decisive fingerprint for locating the transition from single-particle (seniority-type) to collective mode. For the latter purpose, more unambiguous information can be obtained from the evolution of the $E 2$ strengths of seniority-preserving transitions, such as the $4_{1}^{+} \rightarrow 2_{1}^{+}$transition, because it has two distinctive

\footnotetext{
*e-mail: milenas@phys.uni-sofia.bg
}

behaviors in each regime. In seniority regime it follows a parabola with a minimum at the middle of the $j$-shell, while in the collective regime the parabola follows the evolution of the $B\left(E 2 ; 2_{1}^{+} \rightarrow 0_{1}^{+}\right)$strengths, i.e. it has a maximum at the middle of the $j$-shell [2].

Our study is focused on the Po isotopes in the vicinity of ${ }^{208} \mathrm{~Pb}$. The main goal is to derive the absolute $E 2$ strengths for the $4_{1}^{+} \rightarrow 2_{1}^{+}$transitions. This can reveal where and how the transition from seniority type to collective mode occurs. Such study requires the lifetimes of the $4_{1}^{+}$states of ${ }^{206} \mathrm{Po}$ and ${ }^{204} \mathrm{Po}$ to be measured. Until now, such information is missing due to experimental difficulties that stem from the fact that $4_{1}^{+}$states in both isotopes are positioned between the long-lived $8_{1}^{+}$states and the short-lived $2_{1}^{+}$states. In this work we report preliminary results on the $B(E 2)$ transition strengths of the seniorityconserving $4_{1}^{+} \rightarrow 2_{1}^{+}$transitions in ${ }^{204} \mathrm{Po}$ and ${ }^{206} \mathrm{Po}$.

\section{Experiment}

The experiment was performed at the FN Tandem facility at the University of Cologne. Excited states of ${ }^{204}$ Po were populated in the ${ }^{197} \mathrm{Au}\left({ }^{11} \mathrm{~B}, 4 \mathrm{n}\right)$ fusion-evaporation reaction at a beam energy of $55 \mathrm{MeV}$. A thick $\left(110 \mathrm{mg} / \mathrm{cm}^{2}\right)$ self supporting ${ }^{197} \mathrm{Au}$ foil was used as a target. The excited states of ${ }^{206} \mathrm{Po}$ were populated in the ${ }^{198} \mathrm{Pt}\left({ }^{12} \mathrm{C}, 4 \mathrm{n}\right)$ reaction at a beam energy of $65 \mathrm{MeV}$. The used target was $10 \mathrm{mg} / \mathrm{cm}^{2}{ }^{198} \mathrm{Pt}$ foil. The fast-timing array consisted of eight HPGe detectors and nine $\varnothing 1.5^{\prime} \times 1.5^{\prime} \mathrm{LaBr}_{3}(\mathrm{Ce})$ scintillators (referred later in the text as $\mathrm{LaBr}$ ). To suppress the Compton background, six of the $\mathrm{LaBr}$ detec- 
tors were placed inside bismuth-germanate (BGO) Compton suppressors. The other three had lead shields to suppress background events associated with scattered $\gamma$-rays. Time-to-Amplitude Converters (TACs) recorded the time differences between the timing signals for every unique detector-detector combination [3]. To process and collect the energy signals from the detectors and the amplitudes of the TAC signals, $80 \mathrm{MHz}$ synchronized digitizers were used. The data were analyzed using the "soco.v2" software developed at the Institute of Nuclear Physics in Cologne [4]. For the lifetime determination the Generalized Centroid Difference method (GCDM) was used, discussed in detail in [5]. In this method, two independent time spectra are obtained, constructed as the time difference between two signals generated by two $\gamma$-rays that connects an excited state. If the transition which feeds the state provides the start signal to the TAC and the decay transition from this state - the stop signal, the Delayed (D) time distribution is obtained. In the reverse case, Anti-delayed (AD) time distribution is obtained. Assuming no background contributions, the difference between the centroids (first moment of the time distribution) of the delayed and antidelayed time spectra is expressed as :

$$
\Delta C\left(E_{f}, E_{d}\right)=C^{D}-C^{A D}=2 \tau+P R D\left(E_{f}, E_{d}\right),
$$

where $\tau$ is the mean lifetime of the given state and $E_{f}$ and $E_{d}$ are the energies of the feeding and the decaying transition respectively. In this formula, PRD is the prompt response difference, defined as the linearly combined zerotime response of the whole fast-timing array [6]. The PRD is used as a single correction for the lifetime determination, according to Eq. (1), and one of the main tasks is to determine its energy dependence. For calibration of the $\mathrm{PRD},{ }^{152} \mathrm{Eu}$ source has been used. It produces coincident $\gamma$-rays in the $40-1408 \mathrm{keV}$ energy region, which corresponds to the energy region of interest. The lifetimes of the relevant excited states in the daughters ${ }^{152} \mathrm{Gd}$ and ${ }^{152} \mathrm{Sm}$ are known precisely and nearly no background contribution is present in the coincidence spectrum. In Figure 1, $\mathrm{LaBr}$ spectrum is obtained in coincidence with the decay transition from $2_{1}^{+}$to g.s. in ${ }^{152} \mathrm{Gd}$. Time-difference spectra of the 779-344 keV cascade are shown along with the corresponding centroid difference, PRD, peak-to-background (p/b) ratio and lifetime value. Using Eq. (1) the PRD was obtained. Repeating the same procedure for the rest of the feeding transitions of $344 \mathrm{keV}$ state, data points for PRD were obtained. For precise calibration over larger energy region, multiple $\gamma_{\text {feeder }}-\gamma_{\text {decay }}$ combinations were used. [6]

The final PRD data points are fitted using the function

$$
\operatorname{PRD}\left(E_{\gamma}\right)=\frac{a}{\sqrt{e E_{\gamma}^{2}+b}}+c E_{\gamma}+d
$$

The final result of the PRD-curve is presented in Figure 2. The precision is defined as two times the standard rootmean-squared deviation $(2 \sigma)$ of the PRD fit corresponding to 8 ps.

Full projections of the symmetric $\gamma-\gamma$ matrices obtained with the HpGe detectors (red spectrum) and the

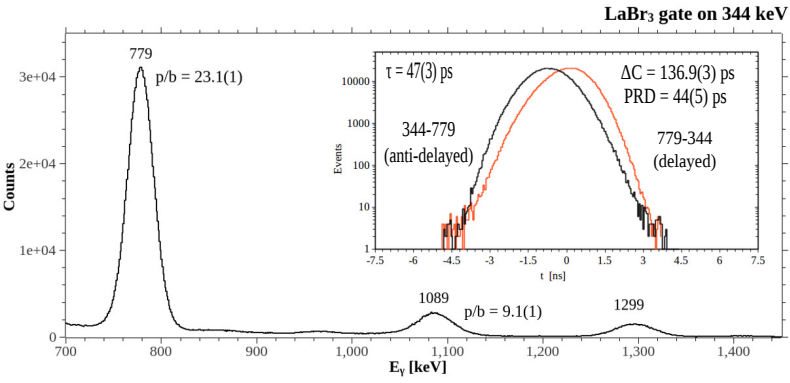

Figure 1. Coincidence $\gamma$-ray spectrum of the $\mathrm{LaBr}$ detector obtained by gating on the $344 \mathrm{keV}$ transition of ${ }^{152} \mathrm{Gd}$. FEP's with no background are obtained at 779, 1089 and $1299 \mathrm{keV}$. Timedifference spectra from the 779-344 keV cascade are shown with the corresponding centroid difference, PRD and lifetime.

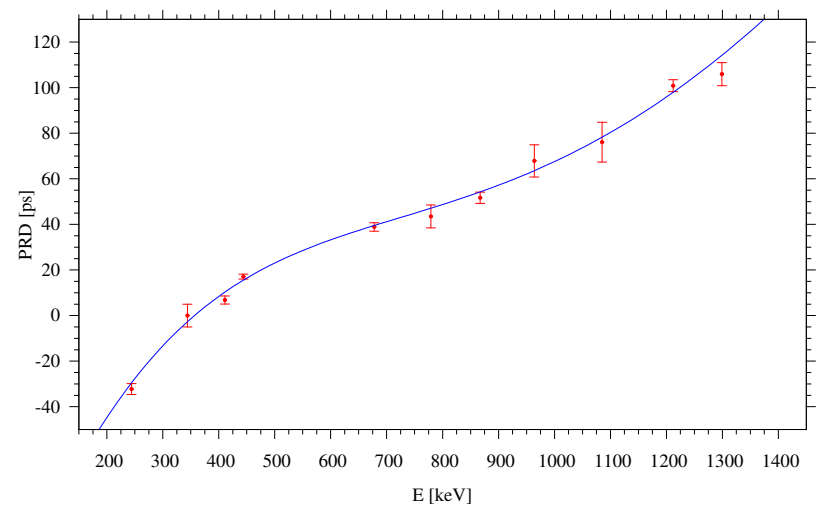

Figure 2. PRD curve obtained using ${ }^{152} \mathrm{Eu}$ source.

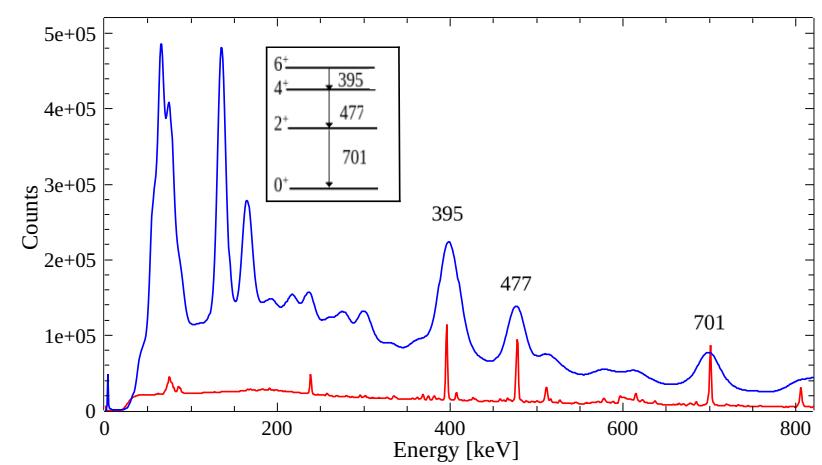

Figure 3. (Color online) Full projections of the symmetric $\gamma$ $\gamma$ matrices obtained with $\mathrm{HpGe}$ detectors(red) and $\mathrm{LaBr}$ detectors(blue) for the case of ${ }^{206} \mathrm{Po}$. The inset shows the relevant partial level scheme of ${ }^{206} \mathrm{Po}$.

$\mathrm{LaBr}$ detectors (blue spectrum) are shown in Figure 3. Partial level scheme of ${ }^{206} \mathrm{Po}$, relevant for the analysis, is also shown on the picture.

To extract the lifetime, triple $\mathrm{HpGe}-\mathrm{LaBr}-\mathrm{LaBr}$ coincidences were used. The doubly gated $\mathrm{HpGe}$ and $\mathrm{LaBr}$ spectra, relevant for the analysis of the $4_{1}^{+}$state in ${ }^{206} \mathrm{Po}$ are shown in Figure 4. The doubly gated HpGe spectrum is generated from $\mathrm{HpGe}-\mathrm{LaBr}-\mathrm{HpGe}$ triple coincidences. The good energy resolution of the HpGe detectors allows a precise coincidence cascade selection. By placing the first $\mathrm{LaBr}$ gate on the $477 \mathrm{keV}$ decay transition, the de- 
Table 1. Lifetimes determined from the fast-timing experiments on ${ }^{204} \mathrm{Po}$ and ${ }^{206} \mathrm{Po}$ and energies of the corresponding gates to obtain time-difference spectra as well as reduced transition probabilities of yrast states in both nuclei, calculated from the measured lifetimes.

\begin{tabular}{cccccccc}
\hline Nucleus & State & $\mathrm{E}_{f}[\mathrm{keV}]$ & $\mathrm{E}_{d}[\mathrm{keV}]$ & HpGe gate $[\mathrm{keV}]$ & $\tau[\mathrm{ps}]$ & transition & $B(E 2)[$ W.u.] \\
\hline${ }^{204} \mathrm{Po}$ & $4_{1}^{+}$ & 426 & 516 & 684 & $23.3(61)$ & $4_{1}^{+} \rightarrow 2_{1}^{+}$ & $13.0(3.4)$ \\
& $2_{1}^{+}$ & 426 & 684 & 516 & $\leqslant 17$ & $2_{1}^{+} \rightarrow 0_{1}^{+}$ & $\geqslant 4.4$ \\
\hline${ }^{206} \mathrm{Po}$ & $4_{1}^{+}$ & 395 & 477 & 701 & $89(12)$ & $4_{1}^{+} \rightarrow 2_{1}^{+}$ & $4.97(40)$ \\
& $2_{1}^{+}$ & 395 & 701 & 477 & $\leqslant 16$ & $2_{1}^{+} \rightarrow 0_{1}^{+}$ & $\geqslant 4.21$ \\
\hline
\end{tabular}

layed and anti-delayed time distributions were obtained, presented in Figure 4. Measuring the difference between the centroids of these time distributions and using Eq. (1), the lifetime can be determined. However, time correction has to be applied, related to the contribution of the timecorrelated Compton background underneath the two full energy peaks (FEP's) of the $\gamma_{\text {feeder }}-\gamma_{\text {decay }}$ cascade. Due to this, $\Delta C_{\text {exp }}$ is corrected with this formula [7]

$\Delta C_{F E P}=\Delta C_{e x p}+\frac{1}{2}\left[\left(\frac{\Delta C_{e x p}-\Delta C_{B G}}{p / b}\right)_{f}+\left(\frac{\Delta C_{e x p}-\Delta C_{B G}}{p / b}\right)_{d}\right]$

where $\triangle C_{F E P}$ corresponds to the centroid difference related to FEP events only. $\Delta C_{B G}$ is the time response of the background and $\mathrm{p} / \mathrm{b}$ is the peak-to-background ratio of the considered $\gamma$-ray. As $\Delta C_{B G}$ cannot be measured directly, it has to be interpolated from measuring background time spectra, generated at different energies above and below the FEP. The analysis to derive the correction for background contributions is performed for each FEP separately. These corrections are presented on the lower two panels of Figure 4. The result for the lifetime of the $4_{1}^{+}$state is given in Table 1 .

The same analysis was carried out for the second nucleus ${ }^{204} \mathrm{Po}$. Full projections of the corresponding $\gamma-\gamma$ matrices obtained with $\mathrm{HpGe}$ detectors and $\mathrm{LaBr}$ detectors are illustrated in Figure 5, along with corresponding level scheme.

Along with the value for the lifetime of $4_{1}^{+}$state in ${ }^{204} \mathrm{Po}$, given in Table 1, an upper limit for the lifetime of the $2_{1}^{+}$state was measured. The $\mathrm{HpGe}$ gate has been placed on the $516 \mathrm{keV}$ transition, while $\mathrm{LaBr}$ gates were placed on the $426 \mathrm{keV}$ and $684 \mathrm{keV}$ transitions respectively. Thus, a summed lifetime for the $4_{1}^{+}$and $2_{1}^{+}$states together was obtained and evaluated to 31.4(64) ps. Subtracting from this value the previously measured value for the lifetime of $4_{1}^{+}$state, an upper limit of $17 \mathrm{ps}$ is deduced.

Using the measured lifetimes, the reduced transition probabilities $B(E 2)$ for corresponding transitions in both nuclei are derived. The results from the analysis are summarized in Table 1.

Figure 6 shows the graphical representation of the $B(E 2)$ values for the case of $N=122$ isotones. It can be seen that the evolution of the $E 2$ strengths for the $4_{1}^{+} \rightarrow 2_{1}^{+}$ transition follows the one for the $2_{1}^{+} \rightarrow 0_{1}^{+}$which rises up towards mid-shell. This indicates that the $4_{1}^{+}$state of ${ }^{206} \mathrm{Po}$ has a collective character. The same situation is observed for ${ }^{204} \mathrm{Po}$ (not shown). These conclusions are in agreement with the conclusion from Ref. [1] that the transition to collectivity occurs before ${ }^{204} \mathrm{Po}$. At the same time, the behavior of the $B\left(E 2 ; 8_{1}^{+} \rightarrow 6_{1}^{+}\right)$in $N=122$ isotonic chain
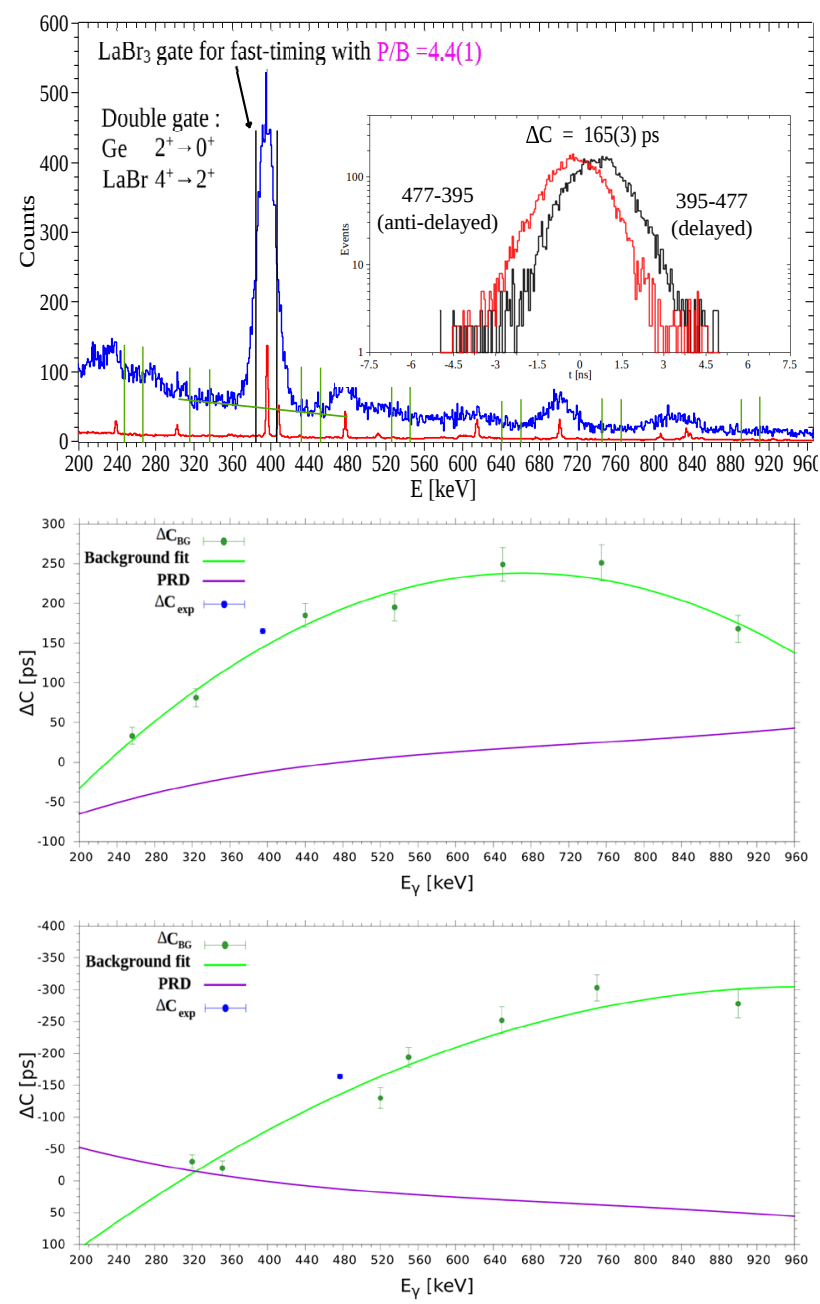

Figure 4. (Color online) Full analysis for extracting the lifetime of the $4_{1}^{+}$state in ${ }^{206} \mathrm{Po}$. Upper panel: Double-gated $\mathrm{HpGe}$ (red) spectra and $\mathrm{LaBr}$ (blue) projection in $\mathrm{HpGe}-\mathrm{LaBr}$ gates, where the first $\mathrm{LaBr}$ gate is placed on the decay transition of the level of interest. Vertical lines indicate ranges of the second $\mathrm{LaBr}$ gate. The obtained time-difference spectra are also shown, with the corresponding value for the centroid difference. Middle and bottom panels show the Compton background correction procedure. Here, the PRD curve is shifted in parallel in order to cross the energy axis at the energy of the decay transition (middle panel) and feeding transition (bottom panel) respectively. 
has a seniority character. All together, this strongly suggests that the transition from seniority regime to collective mode has a spin dependence. However, theoretical calculations are needed to validate this observation.

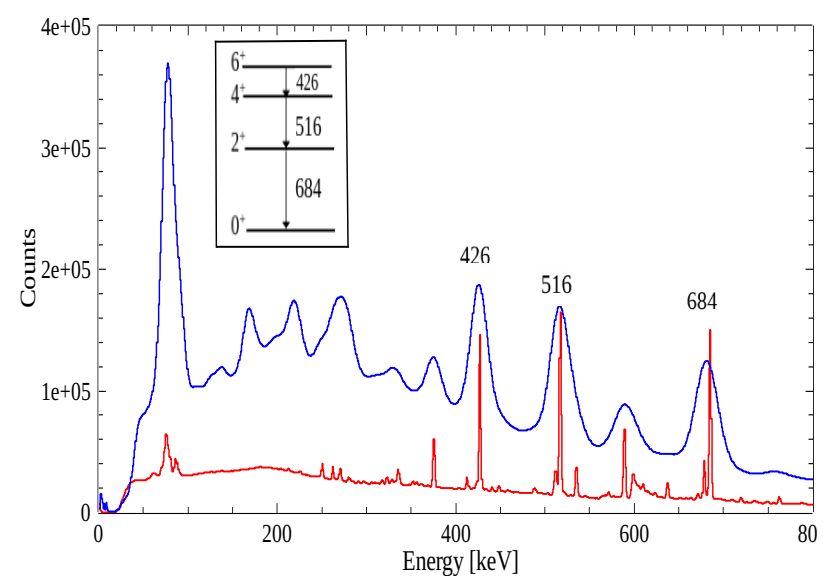

Figure 5. (Color online) Full projections of the symmetric $\gamma-\gamma$ matrices obtained with $\mathrm{HpGe}$ detectors (red) and $\mathrm{LaBr}$ detectors (blue) for the case of ${ }^{204} \mathrm{Po}$. The inset shows the relevant partial level scheme of ${ }^{204} \mathrm{Po}$.

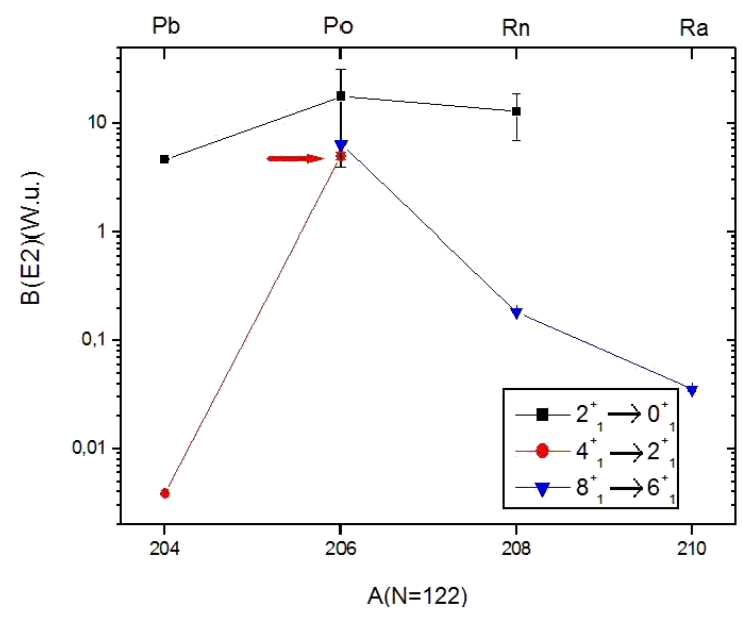

Figure 6. (Color online) Evolution of the $B(E 2)$ values in the $N=122$ isotonic chain. The data have been taken from the present work (corresponding value for the $4_{1}^{+} \rightarrow 2_{1}^{+}$transition), from [1], and references therein.

\section{Conclusion}

We have measured the lifetimes of low-lying yrast states in ${ }^{204} \mathrm{Po}$ and ${ }^{206} \mathrm{Po}$ using a $\mathrm{HpGe}$-gated picosecond-sensitive fast-timing technique. The lifetimes of the $4_{1}^{+}$states in both nuclei were determined, along with a deduced upper limit for the $2_{1}^{+}$state in ${ }^{204}$ Po using GCDM. The evolution of the $B(E 2)$ strengths for the $2_{1}^{+} \rightarrow 0_{1}^{+}$and the $4_{1}^{+} \rightarrow 2_{1}^{+}$ transitions of ${ }^{206} \mathrm{Po}$ and ${ }^{204} \mathrm{Po}$ indicates that these states are of collective nature.

This work was supported by the partnership agreement between the University of Cologne and University of Sofia and by the Bulgarian National Science Fund under Grant No. DN08/23/2016.

\section{References}

[1] T. Grahn et al., Eur. Phys. J. A 52, 340 (2016)

[2] J.J. Ressler, R.F. Casten et al., Phys. Rev. C 69, 034317 (2004)

[3] J.-M. Régis et al., Nucl. Inst. Meth. Phys. Res. A 823, 72 (2016)

[4] J.-M. Régis et al., Nucl. Instrum. Methods Phys. Res. A 763, 210 (2014)

[5] J.-M. Régis et al., Nucl. Instr. Meth. Phys. Res. A 726, 191 (2013)

[6] J.-M. Régis et al., Nucl. Instr. Meth. Phys. Res. A 684, 36 (2012)

[7] J.-M. Régis et al., Phys. Rev. C 95, 054319 (2017) 